\title{
Implicit Active Contours Driven by Local and Global Image Fitting Energy for Image Segmentation and Target Localization
}

\author{
Xiaosheng Yu, Yuanchen Qi, Ziwei Lu, and Nan Hu \\ College of Information Science \& Engineering, Northeastern University, Shenyang 110819, China \\ Correspondence should be addressed to Xiaosheng Yu; yuxiaosheng7@163.com
}

Received 29 August 2013; Accepted 22 October 2013

Academic Editor: Long Cheng

Copyright (c) 2013 Xiaosheng Yu et al. This is an open access article distributed under the Creative Commons Attribution License, which permits unrestricted use, distribution, and reproduction in any medium, provided the original work is properly cited.

We propose a novel active contour model in a variational level set formulation for image segmentation and target localization. We combine a local image fitting term and a global image fitting term to drive the contour evolution. Our model can efficiently segment the images with intensity inhomogeneity with the contour starting anywhere in the image. In its numerical implementation, an efficient numerical schema is used to ensure sufficient numerical accuracy. We validated its effectiveness in numerous synthetic images and real images, and the promising experimental results show its advantages in terms of accuracy, efficiency, and robustness.

\section{Introduction}

Image segmentation is one of the most important operations in the fields of image processing and computer vision. In the past two decades, image segmentation techniques have been widely studied and various novel methods have been proposed; especially the active contour models [1], which are based on the theory of surface evolution and geometric flows, have many successful and promising applications. The basic idea of the active contour models is to define dynamic curves which are evolved in an image domain under the constraints of internal forces and external forces. Generally, the internal forces are derived from the curve itself and the external forces are generated from the image. Active contour models are originally presented in terms of a parametric contour with some drawbacks associated with the difficulty in coping with the complex topological changes and poor dependency of parameterization. Later the level set [2] method was introduced to represent the contour, which improves the active contour models to be completely free of these drawbacks. In the level set method, the contour is represented as the zero level set of a higher dimensional function which is usually called a level set function, and hence the motion of the contour can be implicitly shown by the evolution of the level set function. Motivated by the level set method, a large number of researchers focused on the study of the implicit active contour, including the definition of the image-based driven force, the adaptation to different applications, and the numerical stability.

According to the difference of image-based nature constraints, the existing active contours models can be categorized into two types: edge-based models [3-7] and regionbased models [8-13]. The edge-based models, which typically employ image gradient to construct an edge detector to attract the contours toward the desired boundaries of the objects and finally guarantee the contour evolution stopping on these boundaries. An additional balloon force is often introduced into the evolution functions to enlarge the capture range of the force filed and control the contour to shrink or expand. In practice, they have yielded many successful applications to general images with strong object boundaries. However, they may suffer from some terrible problems such as initialization, boundary leakage, and trapping into local minimum [8], which produce undesirable effects for image segmentation.

Region-based active contour models employ the image statistical information inside and outside the contour to construct the constraints derived from the image data to control 
the whole evolution process. Compared with the edge-based models, region-based models have the following advantages in terms of the initial contour localization and the boundary antileakage and antinoise capability. First, region-based models have more freedom for the initialization of the contour and the exterior and interior contours can be detected simultaneously. Second, they are significantly less sensitive to noise and can efficiently segment the images with weak edges or even without edges. One of the most popular region-based models is the $\mathrm{C}-\mathrm{V}$ model, which has been widely applied to binary phase segmentation [9] with the assumption that images only have two regions and each region is completely homogeneous. However, the $\mathrm{C}-\mathrm{V}$ model fails to segment the images with intensity inhomogeneity.

In order to overcome the considerable segmentation difficulties caused by intensity inhomogeneity, Zhang et al. [12] proposed a local image fitting (LIF) model. The LIF model utilizes the local image information and hence is able to provide desired results. However, the LIF model is sensitive to the initialization of contour, which limits its practical applications.

In this paper, we propose a novel region-based active contour model that has better segmentation performance in the presence of intensity inhomogeneity. We introduce a global image fitting term into the energy functional of the LIF model, which significantly improves the LIF model to be robust with the initial contour being anywhere in the image. An efficient numerical schema is used to ensure sufficient numerical accuracy. The experimental results show its advantages in terms of accuracy, efficiency, and robustness.

This paper is organized as follows. Section 2 introduces the classic C-V and LIF models. Section 3 presents the formulation and implementation of the proposed model. Section 4 validates the proposed model by extensive experiments on synthetic and real images. Section 5 concludes the paper.

\section{C-V and LIF}

2.1. C-V Model. Chan and Vese [8] presented an active contour model to solve the Munford-Shah problem with a special assumption that the input image is piecewise constant. They defined the following energy functional:

$$
\begin{aligned}
E^{\mathrm{CV}}\left(C, c_{1}, c_{2}\right)= & \mu \cdot \text { length }(C)+v \cdot \text { area (inside }(c)) \\
& +\lambda_{1} \int_{\text {inside }(C)}\left|I-c_{1}\right|^{2} d x \\
& +\lambda_{2} \int_{\text {outside }(C)}\left|I-c_{2}\right|^{2} d x
\end{aligned}
$$

where $\mu, v, \lambda_{1}$, and $\lambda_{2}$ are fixed nonnegative parameters. $c_{1}$ and $c_{2}$ are two constants that approximate the image intensities of the regions inside and outside the contour $C$, respectively. Representing the energy functional with a level set formulating and minimizing it, we obtain the following variational formulation:

$$
\begin{aligned}
\frac{\partial \phi}{\partial t}=\delta(\phi)( & \mu \operatorname{div}\left(\frac{\nabla \phi}{|\nabla \phi|}\right)-v \\
& \left.-\lambda_{1}\left(I-c_{1}\right)^{2}+\lambda_{2}\left(I-c_{2}\right)^{2}\right)
\end{aligned}
$$

where $\phi$ is the level set function and $c_{1}$ and $c_{2}$ are given by

$$
\begin{gathered}
c_{1}(\phi)=\frac{\int_{\Omega} I(x) H(\phi) d x}{\int_{\Omega} H(\phi) d x}, \\
c_{2}(\phi)=\frac{\int_{\Omega} I(x)(1-H(\phi)) d x}{\int_{\Omega}(1-H(\phi)) d x} .
\end{gathered}
$$

$-\lambda_{1}\left(I-c_{1}\right)^{2}+\lambda_{2}\left(I-c_{2}\right)^{2}$ is the global image fitting force, which uses the global statistical information of the input image to guide the contour propagation. $\mathrm{C}-\mathrm{V}$ model has an important property that it is less sensitive to the initial contour. However, if the image intensities are inhomogeneity, $c_{1}$ and $c_{2}$ will be far different from the real image data. Hence, the C-V model fails to provide a desired segmentation result.

2.2. LIF Model. Zhang et al. [12] proposed a region-based active contour model which considers the local image information and achieves promising results. They defined the following energy functional:

$$
E^{\mathrm{LIF}}\left(\phi, m_{1}, m_{2}\right)=\frac{1}{2} \int_{\Omega}\left|I-I^{\mathrm{LFI}}\right|^{2} d x
$$

where $I^{\mathrm{LFI}}$ is a local fitted image formulation which can be defined as follows:

$$
I^{\mathrm{LFI}}=m_{1} H(\phi)+m_{2}(1-H(\phi))
$$

where $m_{1}$ and $m_{2}$ are defined by

$$
\begin{aligned}
& m_{1}=\operatorname{mean}\left(I \in\left(\{x \in \Omega \mid \phi(x)<0\} \cap W_{k}(x)\right)\right), \\
& m_{2}=\operatorname{mean}\left(I \in\left(\{x \in \Omega \mid \phi(x)>0\} \cap W_{k}(x)\right)\right),
\end{aligned}
$$

where $W_{k}(x)$ is a rectangular window function such as a truncated Gaussian window or a constant window. $m_{1}$ and $m_{2}$ approximate the weighted averages of the image intensities in a window inside and outside the contour, respectively. Minimizing the energy functional by using the steepest descent method, we derive the gradient descent flow:

$$
\frac{\partial \phi}{\partial t}=\delta(\phi)\left(I-I^{\mathrm{LFI}}\right)\left(m_{1}-m_{2}\right)
$$


In the above functions, $H(\phi)$ is the regularized Heaviside function and $\delta(\phi)$ is the corresponding Dirac function; they are expressed as

$$
\begin{gathered}
H_{\varepsilon}(z)=\frac{1}{2}\left(1+\frac{2}{\pi} \arctan \left(\frac{z}{\varepsilon}\right)\right), \\
\delta_{\varepsilon}(z)=\frac{1}{\pi} \cdot \frac{\varepsilon}{\varepsilon^{2}+z^{2}},
\end{gathered}
$$

where $\varepsilon$ is a positive constant. The local fitted image is constructed by the intensity of points in a rectangular window. This localization property plays a decisive role in segmenting the images with intensity inhomogeneity. The local image fitting force $\left(I-I^{\mathrm{LFI}}\right)\left(m_{1}-m_{2}\right)$ controls the contour evolution. Obviously, when the contour is located in some positions where $m_{1}=m_{2}$, the local image fitting force will be zero, which causes the evolution be trapped into local minima. Consequently, the segmentation result is completely relied on the initialization of the contour.

\section{The Proposed Model}

3.1. The Formulation of the Proposed Model. Motivated by the $\mathrm{C}-\mathrm{V}$ model and LIF model, we define the following energy functional:

$$
\begin{aligned}
& E^{\mathrm{LGIF}}\left(\phi, m_{1}, m_{2}, c_{1}, c_{2}\right) \\
& =(1-a) \frac{1}{2} \int_{\Omega}\left|I-I^{\mathrm{LFI}}\right|^{2} d x \\
& +a\left(\lambda_{1} \int_{\Omega}\left|I-c_{1}\right|^{2} H(\phi) d x\right. \\
& \left.\quad+\lambda_{2} \int_{\Omega}\left|I-c_{2}\right|^{2}(1-H(\phi)) d x\right),
\end{aligned}
$$

where $a$ is a positive constant with $0 \leq a \leq 1$. In order to regularize the zero level set, we need the length of the zero level set, which is defined by

$$
L(\phi)=\int_{\Omega}|\nabla H(\phi(x))| d x .
$$

Now, the entire energy functional can be expressed as

$$
F\left(\phi, m_{1}, m_{2}, c_{1}, c_{2}\right)=E^{\mathrm{LGIF}}+\omega L(\phi),
$$

where $\omega>0$ is a constant as the weight of the length term $L(\phi)$.

Minimizing the energy functional $F\left(\phi, m_{1}, m_{2}, c_{1}, c_{2}\right)$ with respect to $\phi$ by using the steepest descent method, we derive the gradient descent flow:

$$
\frac{\partial \phi}{\partial t}=\omega \delta(\phi) \operatorname{div}\left(\frac{\nabla \phi}{|\nabla \phi|}\right)+\delta(\phi)\left(d_{1}+d_{2}\right)
$$

where

$$
\begin{gathered}
d_{1}=(1-a)\left(I-I^{\mathrm{LFI}}\right)\left(m_{1}-m_{2}\right), \\
d_{2}=a\left(-\lambda_{1}\left(I-c_{1}\right)^{2}+\lambda_{2}\left(I-c_{2}\right)^{2}\right),
\end{gathered}
$$

where $c_{1}, c_{2}, m_{1}$, and $m_{2}$ are defined in (3), (4), and (7), respectively. It is obvious that in the level set evolution equation (13), the new image fitting force is a linear combination of the global image fitting force from the $\mathrm{C}-\mathrm{V}$ model and the local image fitting force from the LIF model. The significance of this force can be explained as follows. The global image fitting force can make the $\mathrm{C}-\mathrm{V}$ model less sensitive to the initial contour, and the local image fitting force can make the LIF success to segment the images intensity inhomogeneity. We combine these two forces with a proper weight $a$ so as to make the proposed model share the advantages of the C-V and LIF models. Hence, the proposed model can handle the intensity inhomogeneity efficiently, no matter where the initial contour starts in the image.

The parameter a plays a significant role to balance the effect of these two forces, which should be chosen according to the degree of inhomogeneity. If the image is existed serious intensity inhomogeneity, we should select small $a$ in which case the contour evolution is mainly controlled by the local image fitting force. If intensity inhomogeneity is minor, we should select large $a$. Thus, the motion of the contour is primarily dependant on the global image fitting force.

Our model is formulated based on the basic frameworks of the C-V model and LIF model. Obviously, if we set $a$ in (10) to 1 and 0 , it will be the C-V model and LIF model, respectively. However, out model considers both the local and global image information, which makes it possess better segmentation property than the C-V and LIF model.

3.2. The Implementation of the Proposed Model. In the traditional level set methods, the level set function is initialized to be a signed distance function (SDF) [2]. In order to prevent the level set function from becoming too steep or flat, a procedure called reinitialization is applied periodically to enforce the degraded level set function being the SDF. However, this procedure is very time consuming and often causes the undesirable side effect of moving the zero level set away from its original location [5]. Many methods [5, 6, 12, $14,15]$ have been proposed to eliminate the reinitialization procedure, among which the Gaussian Filtering Regularized Level Set (GFRLS) method [12] is one of the most successful methods. The GFRLS method is completely free of the costly reinitialization procedure with a series of simple operations in level set evolution. In this paper, we utilize GFRLS method to implement the proposed model. In practice, the GFRLS method firstly initializes the level set function as a binary function and then applies a Gaussian filter to smooth the level set function after each iteration. In addition, in the level set evolution equation, the traditional regular term $\delta(\phi) \operatorname{div}(\nabla \phi /|\nabla \phi|)$ can be eliminated, and during evolution larger time step can be utilized to speed up the contour 


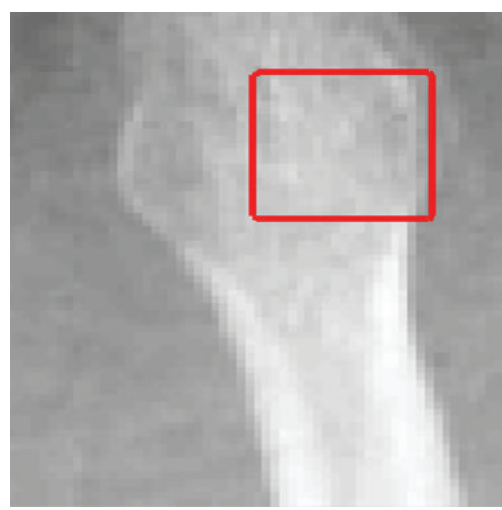

(a)

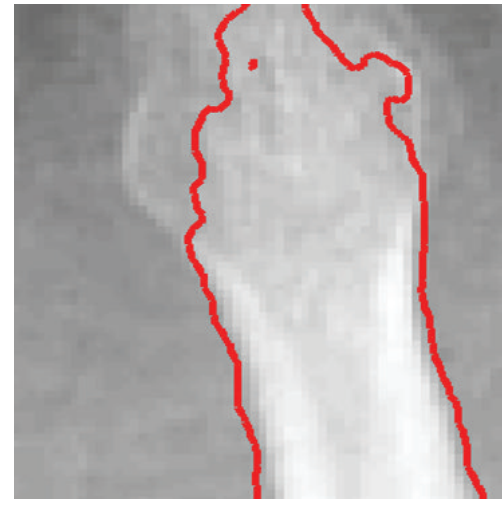

(b)

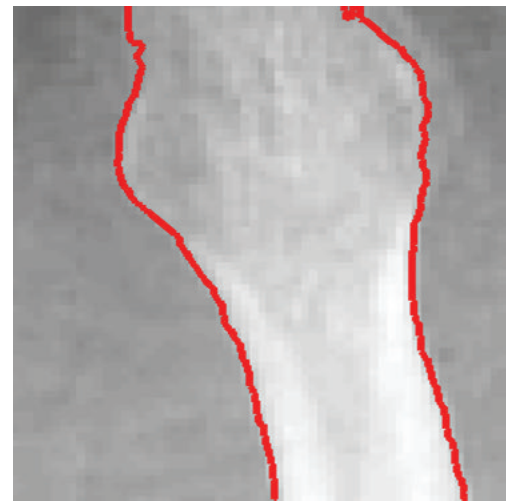

(c)

Figure 1: Comparison of our model with the C-V model in the application to an X-ray image of bone: (a) initial contour, (b) segmentation result of the C-V model, and (c) segmentation result of the our model. The parameters $a=0.05$ and $\sigma=3$.

evolution. Finally, the level set evolution equation of the proposed model can be written as follows:

$$
\frac{\partial \phi}{\partial t}=\delta(\phi)\left(d_{1}+d_{2}\right)
$$
ten as

The approximation iterative equation of (15) can be writ-

$$
\phi^{n+1}=\phi^{n}+\Delta t \delta\left(\phi^{n}\right)\left(d_{1}{ }^{n}+d_{2}{ }^{n}\right),
$$

where $n$ is the iteration number and $\Delta t$ is the time step. The major steps of the proposed model can be concluded as follows.

(1) Initialize a level set function $\phi$ as

$$
\phi(x)= \begin{cases}-c_{0}, & x \in R, \\ c_{0}, & \text { otherwise }\end{cases}
$$

where $c_{0}$ is a positive constant and $R$ is a region in the image.

(2) Update $c_{1}, c_{2}, m_{1}$, and $m_{2}$ using (3), (4), and (7), respectively.

(3) Update the level set function $\phi$ according to (16).

(4) Regularize the level set function $\phi$ with a Gaussian Kernel, $\phi=G_{\xi} * \phi$, where $\xi$ is the standard deviation.

(5) Return step 2 until the level set function has converged.

\section{Experimental Results}

This section shows the segmentation results of the proposed model for both synthetic and real images of different modalities. All algorithms in the experiments are implemented in Matlab 7.8 on a $2.19-\mathrm{GHz}$ Intel Pentium Dual personal computer. We select a truncated Gaussian window $k_{\sigma}(x)$ with standard deviation $\sigma$ of size $4 k+1$ by $4 k+1$, where $k$ is the greatest integer smaller than $\sigma$. There are parameters $a, \sigma, \lambda_{1}, \lambda_{2}$, and $\varepsilon$ in our model and $c_{0}, \Delta t$, and $\xi$ for the implementation. We use the following fixed parameters: $\lambda_{1}=$ $\lambda_{2}=1, \varepsilon=1, c_{0}=1, \Delta t=0.1$, and $\xi=0.5$. The parameters $a$ and $\sigma$ need to be tuned according to the images.

4.1. Comparisons with the C-V Model. Figures 1 and 2 show the comparisons between $\mathrm{C}-\mathrm{V}$ model and our model to segment an X-ray image of bone and a synthetic image, respectively. We use the same initial contours in both models for fair comparisons and set the parameters $a=0.05, \sigma=3$ and $a=0.1, \sigma=7$. Obviously, intensity inhomogeneity occurs in these two images. It is clearly seen that the C$\mathrm{V}$ model does not work well for them as explained in Section 2.1. By contrast, our model utilizes the local and global image fitting forces to drive the contour evolution and successfully extract the desired objects.

4.2. Comparisons with the LIF Model. We apply our model to segment an infrared image of intensity inhomogeneity shown in Figure 3. We set the parameters $a=0.01$ and $\sigma=$ 5. Figure 3(a) shows the initial contours which are circular with the same radius in different positions of the image. Figures 3(b) and 3(c) show the results by the LIF model and our model, respectively. It can be observed that the LIF model yields an accurate result only when the contour started in the middle of the object. By contrast, our model provides desired segmentation results with all initializations.

Figure 4 illustrates the segmentation results on a CT image of heart by the LIF model and our model. We set the parameters $a=0.03$ and $\sigma=3$. Figure 4(a) shows the square initial contours. Figure 4(b) shows the results by the LIF model. The LIF model fails to segment ventricles with these initializations. Our model achieves the satisfactory results with the same initializations as shown in Figure 4(c).

Figure 5 shows the segmentation results on a vessel image by the LIF model and our model. We set the parameters 


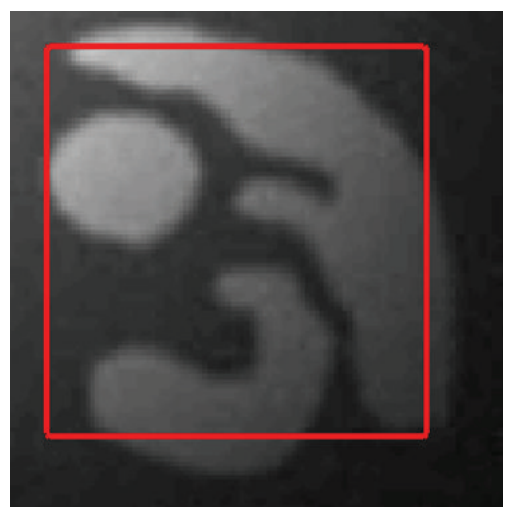

(a)

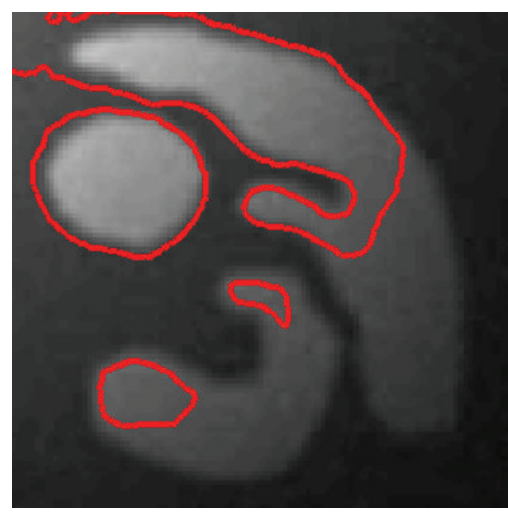

(b)

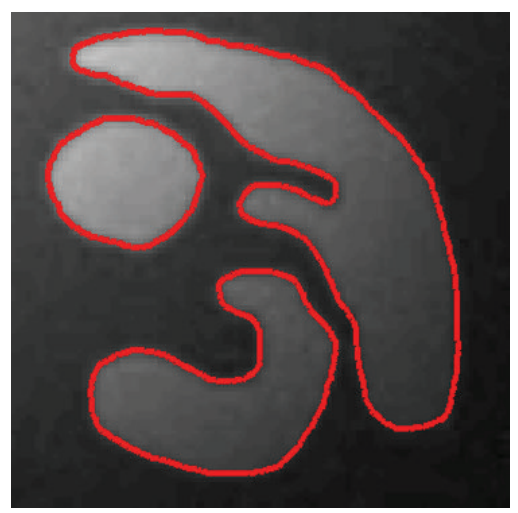

(c)

Figure 2: Comparison of our model with the C-V model in the application to a synthetic image: (a) initial contour, (b) segmentation result of the C-V model, and (c) segmentation result of our model. The parameters $a=0.1$ and $\sigma=7$.
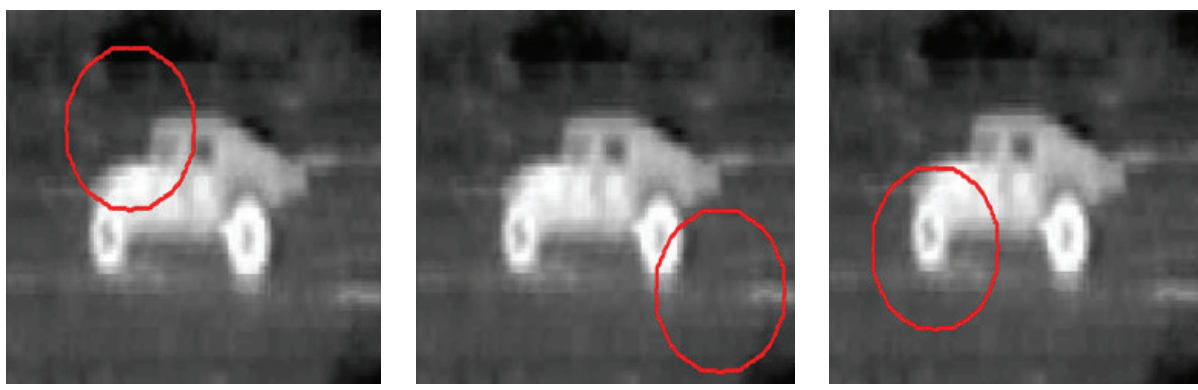

(a)
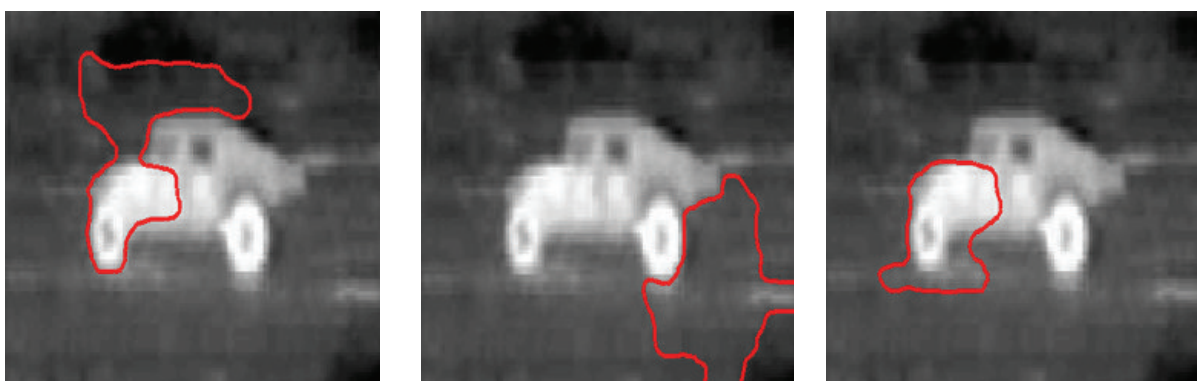

(b)
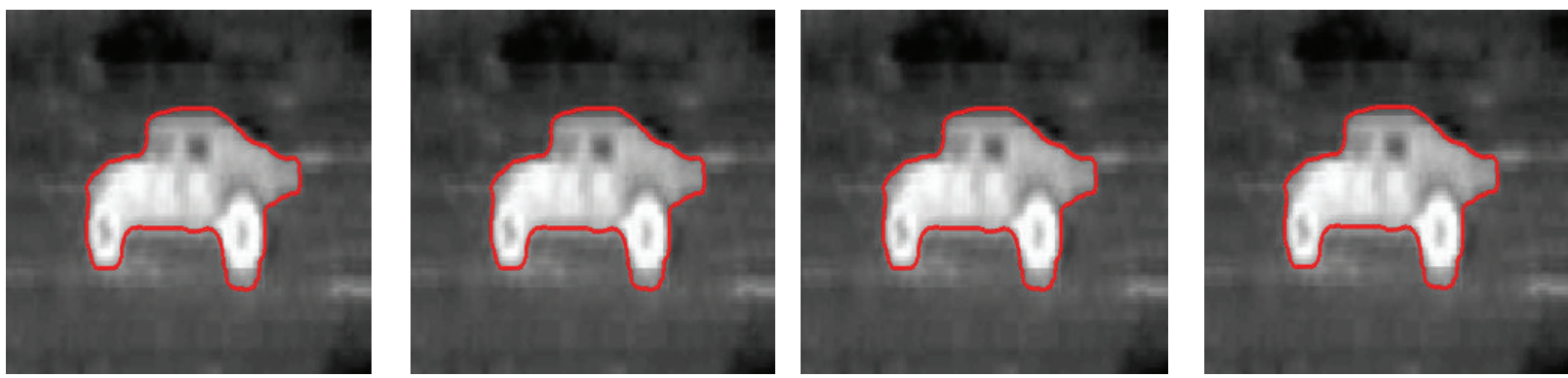

(c)

FIGURE 3: Performances of our model and the LIF model on an infrared image with different initial contours: (a) initial contours, (b) segmentation results of the LIF model, and (c) segmentation results of our model. The parameters $a=0.01$ and $\sigma=5$. 

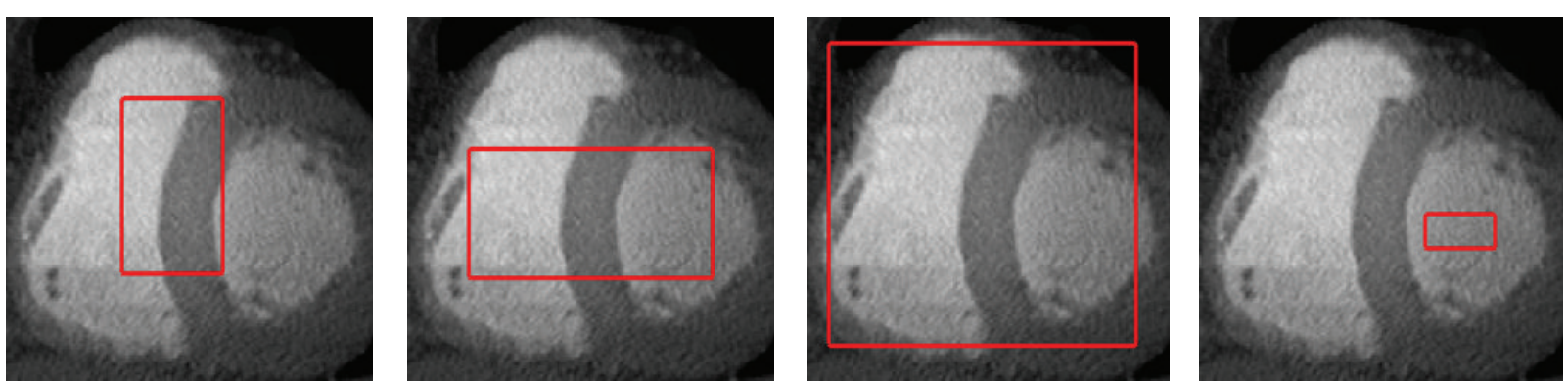

(a)
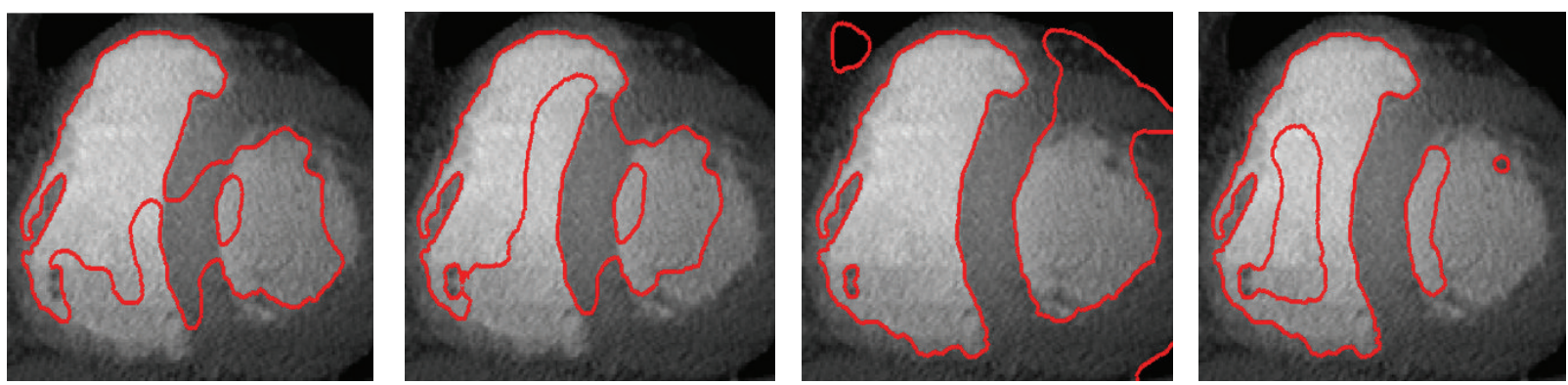

(b)
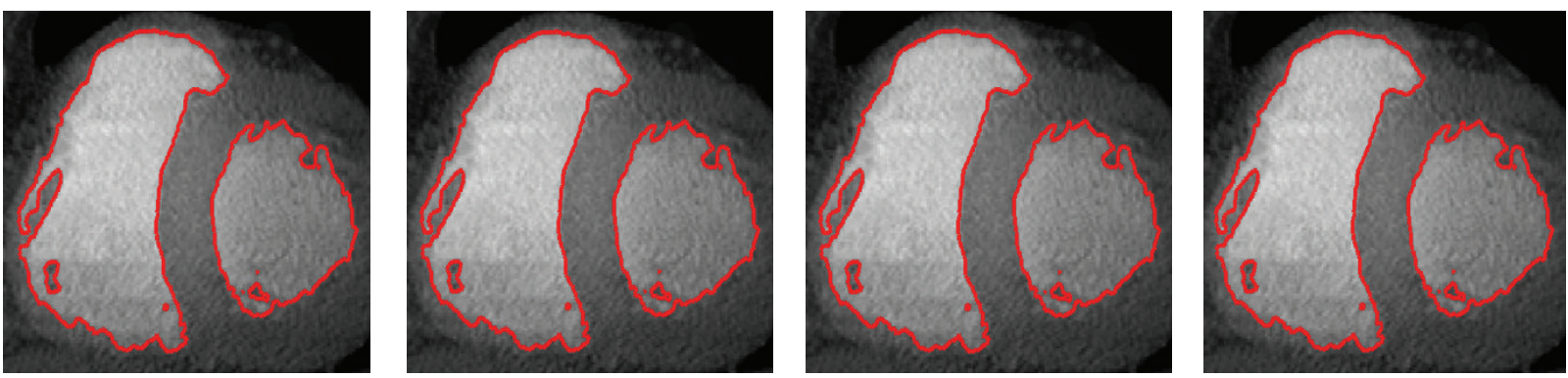

(c)

FIGURE 4: Performances of our model and the LIF model on a CT image of heart with different initial contours: (a) initial contours, (b) segmentation results of the LIF model, and (c) segmentation results of our model. The parameters $a=0.03$ and $\sigma=3$.

$a=0.05$ and $\sigma=7$. The initial contours with different shapes are located around, inside, outside, and across the object, respectively. Figure 4(b) shows the results by the LIF model. Obviously, the LIF successfully extract the desired object only when the contour is outside of the object. Our model always detects the object of interest with the initial contour being anywhere in the image.

The above three experiments demonstrate that our model is robust to the initialization of the contour. In order to quantitatively evaluate the segmentation performance of our model and the LIF model in these three experiments, we utilize the Jaccard similarity (JS) [16] for comparison. JS can be defined by

$$
J\left(S_{1}, S_{2}\right)=\frac{\left|S_{1} \cap S_{2}\right|}{\left|S_{1} \cup S_{2}\right|},
$$

where $S_{1}$ and $S_{2}$ are the real segmentation result and ground truth, respectively. The closer the JS value to 1 , the better the segmentation. In our experiment, we calculate the average of the JS values of our model and LIF model with different initializations. Figure 6 presents the JS values of these two models. It can be seen that our model has better performance with different initial contours.

\section{Conclusion}

This paper presents a novel region-based active contour model in a variational level set formulation. Our model utilizes both the local and global image information to control the motion of the contour, and hence, it can efficiently handle intensity inhomogeneity with flexible initialization. Our model is implemented with GFRLS method, which can eliminate the requirement of reinitialization. Experiment results demonstrated that our model has better performance than the C-V model and LIF model. 

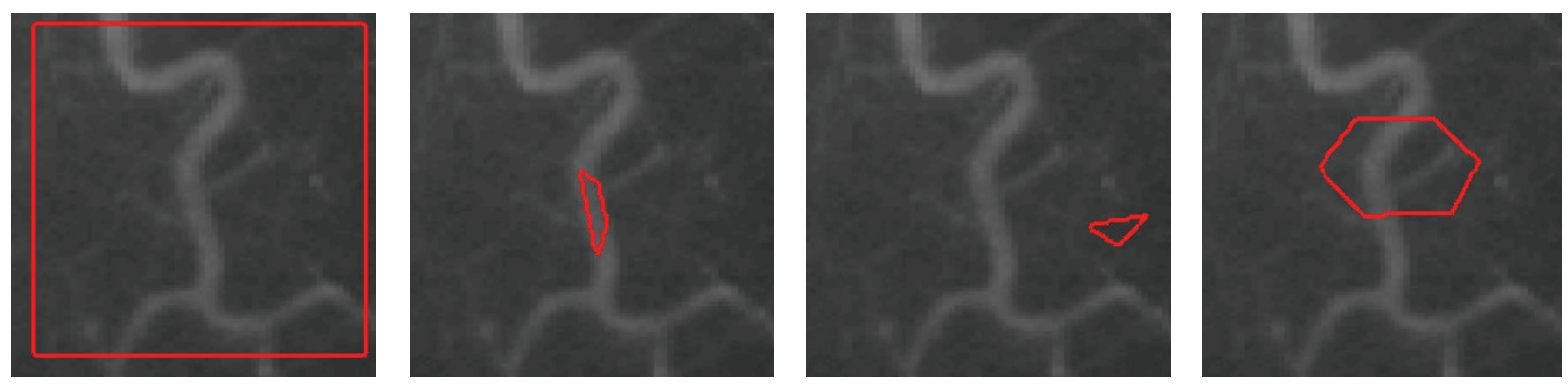

(a)
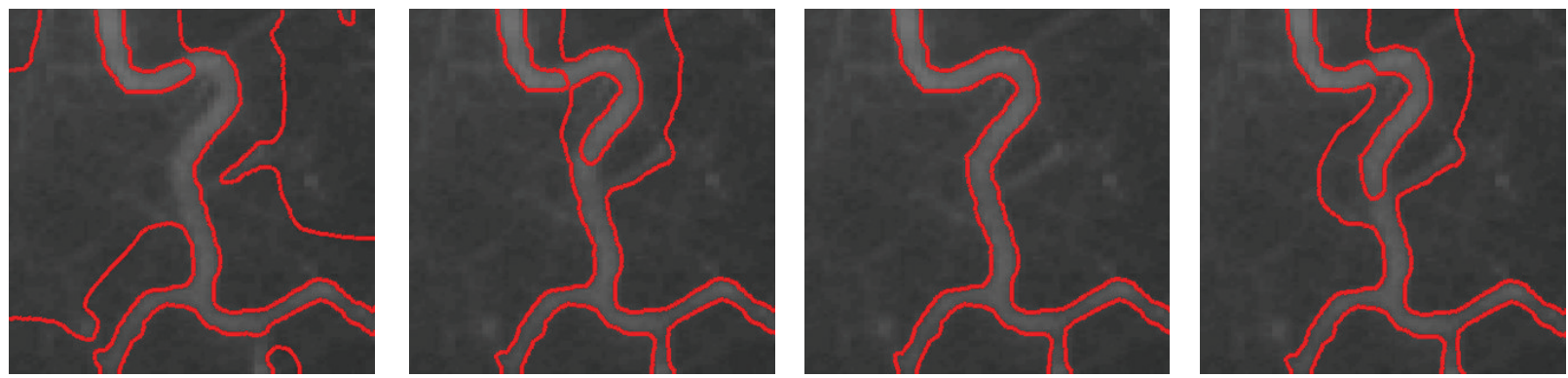

(b)
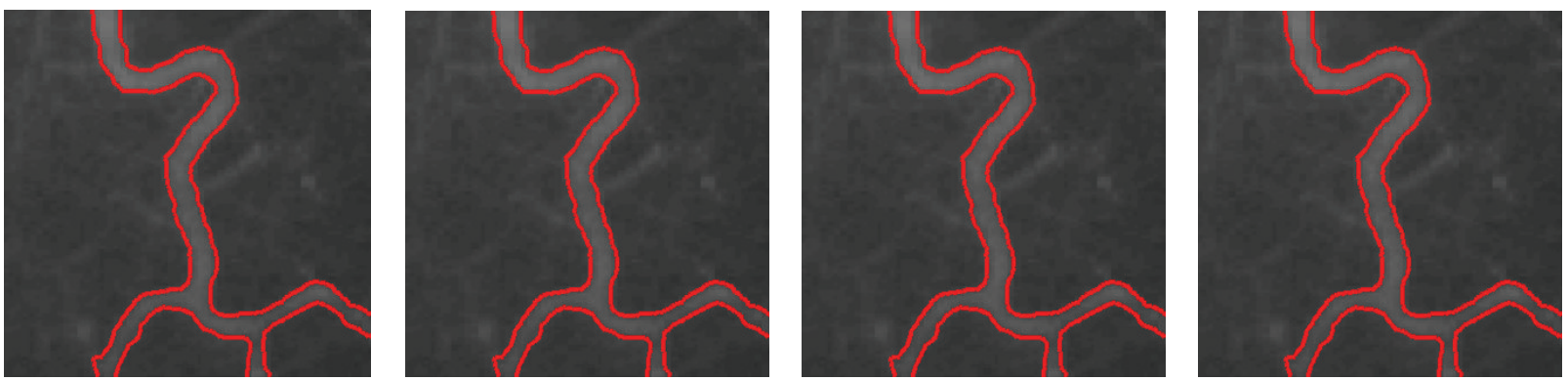

(c)

FIGURE 5: Performances of our model and the LIF model on a vessel image with different initial contours: (a) initial contours, (b) segmentation results of the LIF model, and (c) segmentation results of our model. The parameters $a=0.05$ and $\sigma=7$.

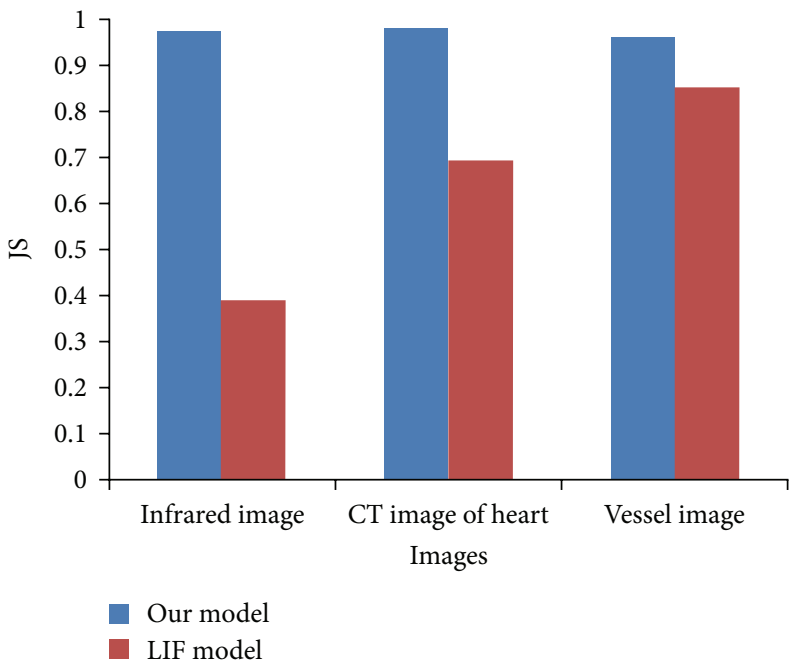

FIgURE 6: Quantitative comparisons between our model and the LIF model. 


\section{Conflict of Interests}

The authors declare that there is no conflict of interests regarding the publication of this paper.

\section{Acknowledgment}

The research is supported by the National Science Foundation of China (61273078), Science Foundation of Liaoning (20102062), and Fundamental Research Funds for the Central Universities (N110604006).

\section{References}

[1] M. Kass, A. Witkin, and D. Terzopoulos, "Snakes: active contour models," International Journal of Computer Vision, vol. 1, no. 4, pp. 321-331, 1988.

[2] S. Osher and J. A. Sethian, "Fronts propagating with curvaturedependent speed: algorithms based on Hamilton-Jacobi formulations," Journal of Computational Physics, vol. 79, no. 1, pp. 1249, 1988.

[3] V. Caselles, R. Kimmel, and G. Sapiro, "Geodesic active contours," International Journal of Computer Vision, vol. 22, no. 1, pp. 61-79, 1997.

[4] N. Paragios, O. Mellina-Gottardo, and V. Ramesh, "Gradient vector flow fast geometric active contours," IEEE Transactions on Pattern Analysis and Machine Intelligence, vol. 26, no. 3, pp. 402-407, 2004.

[5] C. Li, C. Xu, C. Gui, and M. D. Fox, "Level set evolution without re-initialization: a new variational formulation," in Proceedings of the IEEE Computer Society Conference on Computer Vision and Pattern Recognition (CVPR '05), vol. 1, pp. 430-436, June 2005.

[6] C. Li, C. Xu, C. Gui, and M. D. Fox, "Distance regularized level set evolution and its application to image segmentation," IEEE Transactions on Image Processing, vol. 19, no. 12, pp. 3243-3254, 2010.

[7] X. Xie and M. Mirmehdi, "MAC: magnetostatic active contour model," IEEE Transactions on Pattern Analysis and Machine Intelligence, vol. 30, no. 4, pp. 632-646, 2008.

[8] T. F. Chan and L. A. Vese, "Active contours without edges," IEEE Transactions on Image Processing, vol. 10, no. 2, pp. 266-277, 2001.

[9] C. Li, C. Kao, J. C. Gore, and Z. Ding, "Implicit active contours driven by local binary fitting energy," in Proceedings of the IEEE Conference on Computer Vision and Pattern Recognition (CVPR '07), pp. 1-7, Minneapolis, Minn, USA, June 2007.

[10] L. Wang, L. He, A. Mishra, and C. Li, "Active contours driven by local Gaussian distribution fitting energy," Signal Processing, vol. 89, no. 12, pp. 2435-2447, 2009.

[11] C. Li, R. Huang, Z. Ding, J. C. Gatenby, D. N. Metaxas, and J. C. Gore, "A level set method for image segmentation in the presence of intensity inhomogeneities with application to MRI," IEEE Transactions on Image Processing, vol. 20, no. 7, pp. 20072016, 2011.

[12] K. Zhang, H. Song, and L. Zhang, "Active contours driven by local image fitting energy," Pattern Recognition, vol. 43, no. 4, pp. 1199-1206, 2010.

[13] K. Zhang, L. Zhang, and S. Zhang, "A variational multiphase level set approach to simultaneous segmentation and bias correction," in Proceedings of the 17th IEEE International Conference on Image Processing (ICIP '10), pp. 4105-4108, Hong Kong, China, September 2010.

[14] X. Xie, "Active contouring based on gradient vector interaction and constrained level set diffusion," IEEE Transactions on Image Processing, vol. 19, no. 1, pp. 154-164, 2010.

[15] K. Zhang, L. Zhang, H. Song, and W. Zhou, "Active contours with selective local or global segmentation: a new formulation and level set method," Image and Vision Computing, vol. 28, no. 4, pp. 668-676, 2010.

[16] M. Everingham, L. van Gool, C. K. I. Williams, J. Winn, and A. Zisserman, "The pascal visual object classes (VOC) challenge," International Journal of Computer Vision, vol. 88, no. 2, pp. 303338, 2010. 

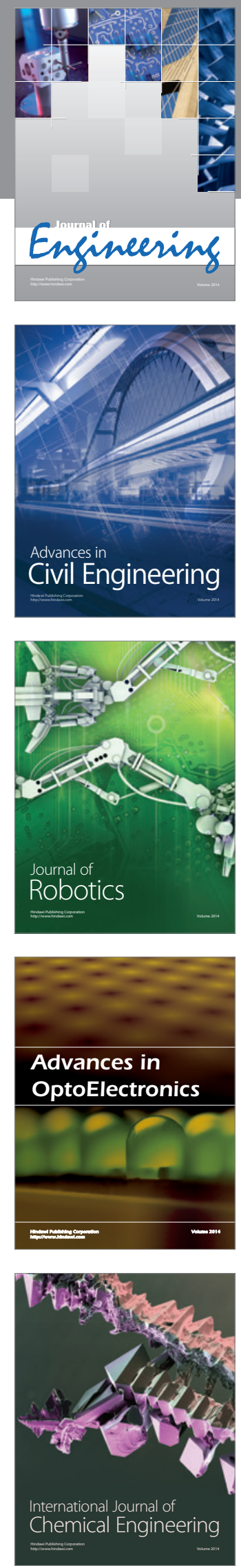

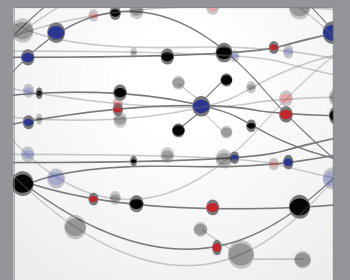

The Scientific World Journal
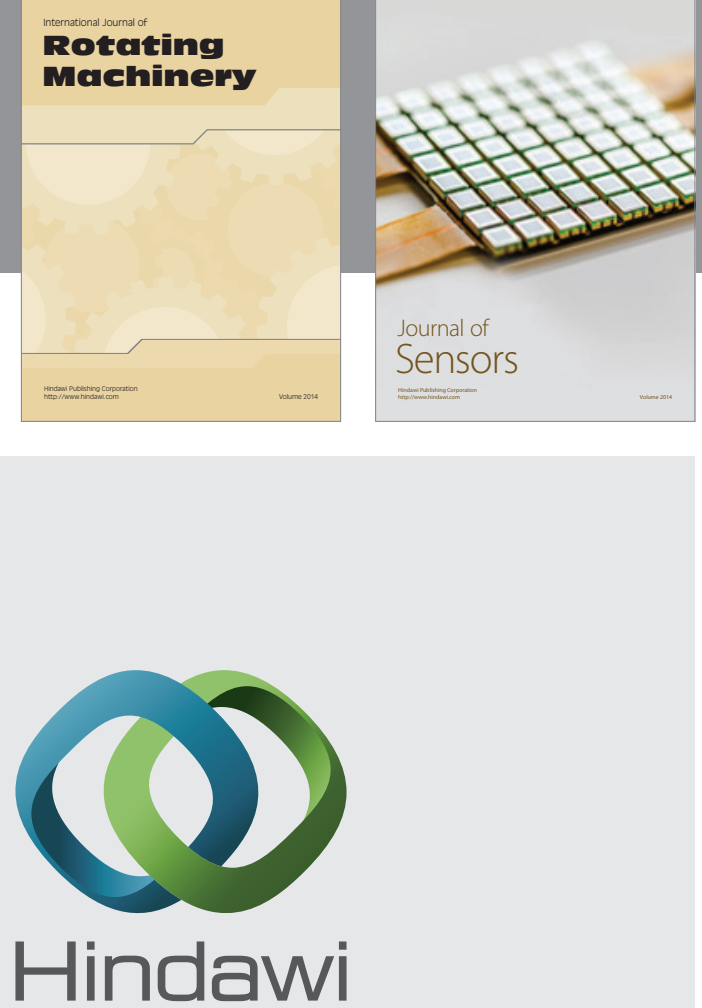

Submit your manuscripts at http://www.hindawi.com
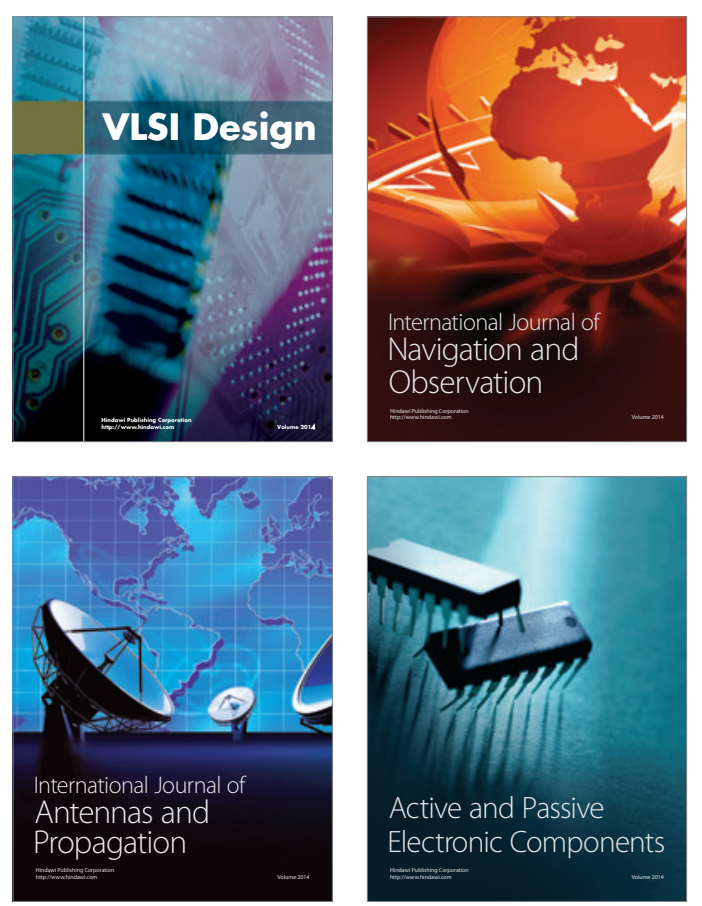
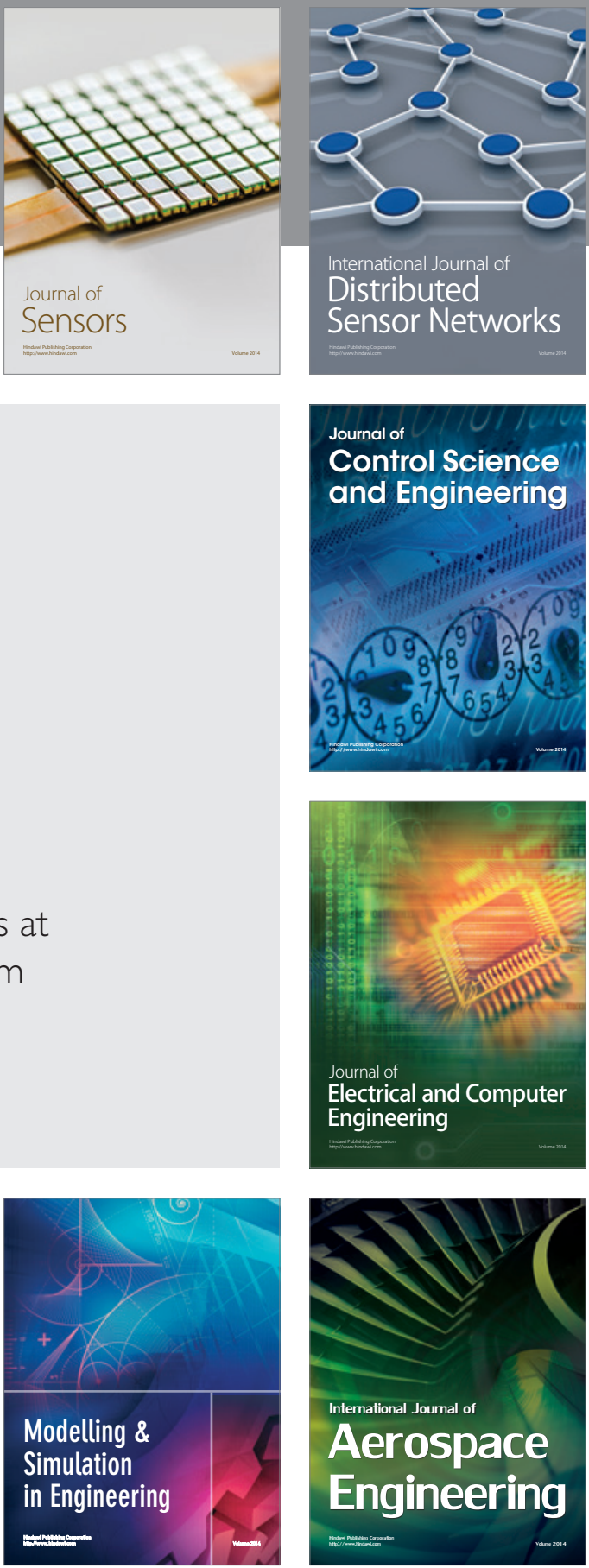

Journal of

Control Science

and Engineering
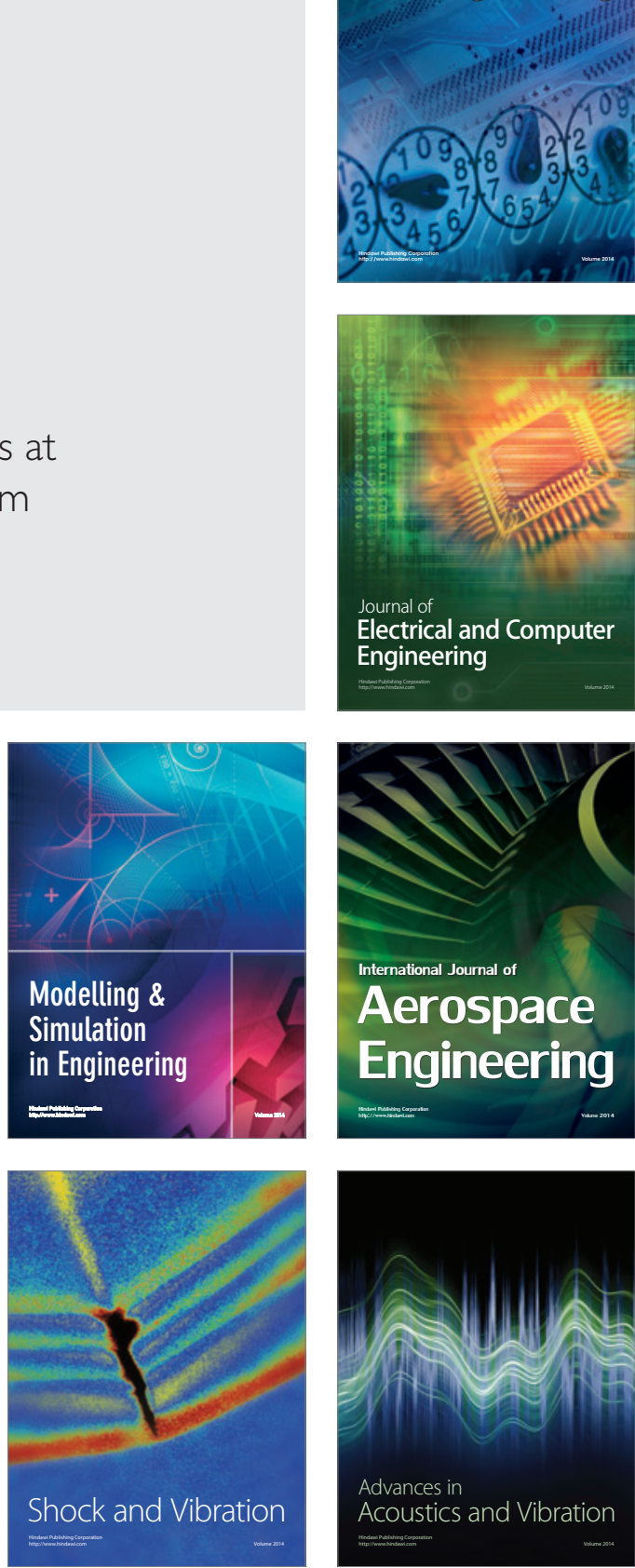